\title{
Rate of elapsed polymerization of hydroxyethylacrylate gel induced by gamma irradiation
}

\begin{abstract}
The rate of elapsed polymerization of polyhydroxyethylacrylate in gelatin has been studied to investigate the effect of co-monomers consumption at a given dose. The polymer gel dosimeters consisted of 2\%-4\% N,N-methyelene-bis-acrylamide cross-linker, $2 \%-4 \% 2$ hydroxyethylacrylate monomer and gelatin at 3\% and 5\%. The dosimeters were irradiated by using $60 \mathrm{Co}$ teletherapy $\gamma$-ray source up to $20 \mathrm{~Gy}$ at a constant dose rate. The relaxation rate of water proton in the dosimeters at different doses and co-monomer concentrations were measured using a nuclear magnetic resonance spectroscopy. The rate of elapsed polymerization decreases with increasing the dose and the initial concentration of comonomers. The rate of consumption of co-monomers increases with an increase of the polymerization and the gelatin content of the polymer gel.
\end{abstract}

Keyword: Elapsed polymerization,Relaxation rate,Consumption rate, Polymer gel dosimeter 